\title{
Sparse MR Image Reconstruction Considering Rician Noise Models: A CNN Approach
}

\author{
M. V. R. Manimala ${ }^{1}$ (D) C. Dhanunjaya Naidu ${ }^{2} \cdot$ M. N. Giri Prasad ${ }^{1}$ \\ Published online: 11 August 2020 \\ (c) Springer Science+Business Media, LLC, part of Springer Nature 2020
}

\begin{abstract}
Compressive sensing (CS) provides a potential platform for acquiring slow and sequential data, as in magnetic resonance (MR) imaging. However, CS requires high computational time for reconstructing MR images from sparse $k$-space data, which restricts its usage for high speed online reconstruction and wireless communications. Another major challenge is removal of Rician noise from magnitude MR images which changes the image characteristics, and thus affects the clinical usefulness. The work carried out so far predominantly models MRI noise as a Gaussian type. The use of advanced noise models primarily Rician type in CS paradigm is less explored. In this work, we develop a novel framework to reconstruct MR images with high speed and visual quality from noisy sparse $k$-space data. The proposed algorithm employs a convolutional neural network $(\mathrm{CNN})$ to denoise MR images corrupted with Rician noise. To extract local features, the algorithm exploits signal similarities by processing similar patches as a group. An imperative reduction in the run time has been achieved as the CNN has been trained on a GPU with Convolutional Architecture for Fast Feature Embedding framework making it suitable for online reconstruction. The CNN based reconstruction also eliminates the necessity of optimization and prediction of noise level while denoising, which is the major advantage over existing state-of-the-art-techniques. Analytical experiments have been carried out with various undersampling schemes and the experimental results demonstrate high accuracy and consistent peak signal to noise ratio even at 20-fold undersampling. High undersampling rates provide scope for wireless transmission of $k$-space data and high speed reconstruction provides applicability of our algorithm for remote health monitoring.
\end{abstract}

Keywords Sparsity · Convolutional neural network - Magnetic resonance imaging · Compressive sensing $\cdot$ Rician noise

M. V. R. Manimala

svrmanimala_ece@mvsrec.edu.in

C. Dhanunjaya Naidu

cdnaidu@vnrvjiet.in

M. N. Giri Prasad

mahendragiri1960@gmail.com

1 JNTUA, Ananthapuramu, India

2 VNRVJIET, Hyderabad, India 


\section{Introduction}

Magnetic resonance imaging (MRI) is a non-invasive medical imaging modality which is widely utilized for scanning physiological changes in tissues and organs of the human body. It is used particularly because it can produce high-quality 3D images. Diffusionweighted MRI (DWMRI) and diffusion tensor MRI (DT-MRI) generates contrast, whereas functional MRI (fMRI) measures changes in blood flow and oxygen levels to evaluate the brain activity. These MRI techniques are widely utilized across the globe for investigation of brain tissues. They exploit the fine disparity inherent between the tissues to provide medical images with high spatial resolution. Despite the high resolution, there are certain limitations associated with MR medical imaging like high processing and relaxation time, high slew rate and slow acquisition [1-3]. Moreover, MR images are highly influenced by the noise from different sources at the time of acquisition, which leads to distortion and unpredictable scanning. This noise and distortion can directly affect the acquisition time, spatial resolution and signal-to-noise ratio (SNR) thereby unbalancing the acquisition mechanism, and hence poses a major challenge for MRI modalities [4]. Denoising MR images is a pre-requisite for attaining higher image quality. Lately, researchers have worked on decreasing the acquisition time, noise and distortion present in the MR images.

Compressive sensing (CS) addresses the problem of slow acquisition which is inherent with MR imaging. This is done by reconstructing high quality images from few $k$-space measurements that are far below the Nyquist Rate [1, 2]. In 2007, Lustig et al. presented compressed sensing magnetic resonance imaging (CSMRI) technique by considering various undersampling schemes [1]. Dictionary learning for MRI (DLMRI) provided an effective solution to recover MR images from sparse $k$-space data [2], but had a drawback of high computational time.

CS achieves high quality of MR image reconstruction by utilizing the transform domain sparsity in the encoded MR acquisitions and thereby adapting non-linear reconstruction. Despite the numerous advantages of CS, there are various issues that need to be sorted, such as high reconstruction time, optimization of sampling trajectories, efficient sparse transforms which are incoherent with sampling operators to achieve clinical reconstruction quality.

Extended version of non local means filter for denoising fully sampled 3D MR images corrupted with Rician noise was implemented in [5]. A regularized sensitivity encoding for Fast MRI (SENSE) [6] reconstruction technique reduced the artifacts present in MR images. An analysis of MR reconstruction methods using a priori information inherent in the compressed sensed MR images has been presented in [7, 8]. Gaussian mixture model in [9], reconstructs signal from noisy sparse measurements. Traditional CS recovery approach demands high computational time, instigates the researcher to explore usage of GPUs for recovering sparse signals. In [10], noise in MR image has been reduced with the high speed GPU acceleration based on trilateral filter. In [11], a brain MR image reconstruction based on sparse representation, gradient information and non-symmetry anti-packing model was introduced to minimize the reconstruction loss and computation time.

Although various techniques prevails for denoising the MR images, the work carried so far chiefly models MRI noise as a Gaussian type. Based on our knowledge, regarding the use of intricate noise models, primarily Rician type was scarcely studied for the reconstruction of MR images from sparse $k$-space data. Asymmetric Rician distribution sets in a signal dependent bias which predominantly affects the image contrast and thus poses a major challenge in denoising [12]. Hence, in this work we present the concept of 
denoising MR images based on sparse representation with CNN methods. The proposed denoising algorithm reconstructs MR images with high visual quality, further; it can be directly employed without optimization and prediction of the Rician noise level. The algorithm reduces artifacts in an effective manner by preserving local structures present in the MR images. Experimental results of the algorithm have been compared with several existing state-of-the-art techniques, such as DLMRI [2], non-local means (NLM) [5] and its variants. Experimental outcomes exhibit significant improvement in the peak signal to noise ratio (PSNR) compared to the existing algorithms which are considered in this paper. Further, the quality of reconstruction has also been assessed with metrics namely, feature similarity index metric (FSIM), high frequency error norm (HFEN) and quantitative index based on local variance (QILV). The algorithm developed by us solves optimization by minimizing the rank iteratively, hence converges very quickly and also provides precise reconstruction from sparse $k$-space data. The above attributes provides potential applicability to our denoising method in remote health monitoring.

The rest of the article is organized as follows. Section 2, discusses the related work on sparse MRI. In Sect. 3, we discuss the methods for solving the sparse optimization problem relating it with the proposed CNN-based formulation. The novel CNN-based Rician denoising model has been detailed in Sect. 4. Experimental results are presented in Sect. 5 and Sect. 6 summarizes and concludes this paper.

\section{Related Work on MRI}

MRI is an excellent imaging modality to diagnose pathological and physiological alterations that occur in the human body, however, the $k$-space data acquisition remains very slow as it is done sequentially in time. Although some increase in the speed has been achieved by employing parallel acquisition of data and multiple RF receiver coils, however, it also introduces noise and aliasing. This initiated various researchers to work towards reducing these problems and some of the related literature is presented below.

The presence of geometric regularities and unpredictable sparse singularities can lead to staircase artifacts. Bandlet basis provided denoising along with compression by dividing the geometrically regular images [3]. Total variation (TV) is one of the popular methods; however it could be employed only when the images had constant regions. In [13], total generalized variation (TGV) gets over this limitation to provide good denoising for images having varying characteristics. Low rank modeling enables constrained recovery of MR images with slow phase variations from limited $k$-space data [14]. It finds potential applicability in dynamic MR imaging. In [15], a reconstruction for diffusion-weighted MR images has been developed which was resilient to motion and could fix phase mismatches. These methods required high computation time.

A breakthrough in the image processing was achieved with the introduction of deep learning methods. Krizhevsky et al. developed a deep CNN for image classification in [16] that gave a new insight to image processing. Deep learning methods employ supervised learning which provides accurate reconstruction at very high speed. Since training the deep networks is laborious, a network known as ResNet was introduced in [17] to overcome this challenge. The layers in ResNet were trained from the residual functions with respect to the input layer rather than learning from unreferenced functions. In [18], a cascaded CNN was introduced to tackle poor SNR of the reconstructed image resulting from sparse diffusion tensor cardiac magnetic resonance images (DTC-MRI). In [19], an adversarial network has 
been employed for perceptual refinement in the reconstructed MR images which provided a better stability to the training model. In [20, 21], a deep learning convolutional neural network (DLCNN) approach using U-Net was introduced for the reconstruction of undersampled MR images. In [21], $k$-space correction was used to eliminate folding artifacts that resulted due to undersampling. Basic U-Net was extended to dual and tight frame U-Net in [22] to provide noise resistant reconstruction from sparse computer tomography (CT) data. In U-Net++ [23], skip connections were utilized to decrease the semantic difference between the feature maps of the encoder and decoder thereby easing the learning process. The U-Net++ was further extended in [24], to provide segmentation of varied sized objects in multiple modalities that included MRI, CT scan and electron microscopy (EM). In [25], a multi-scale 3D dictionary was developed using a variant of alternating direction method of multipliers (ADMM) for dynamic MR image reconstruction along with the regularization method. A deep neural network architecture along with bandpass filtering has been presented in [26] for the efficient reconstruction of MR images. Although a patch based approach enhanced the consistency with data measurements and provided a better accuracy, however the inherent correlation between the patches was not exploited. In [27] variational autoencoder (VAE) has been utilized for reconstruction of MR images by learning the distribution of image patches. Acquisition time and artifacts due to coherent aliasing has been reduced through deep residual learning from sparsely sampled $k$-space data in [28].

In [29], CNN was employed for left ventricle segmentation and removal of the outliers in the cardiac MRI. In [30], ResNet [17] was employed for accurate T1 mapping with very low error as compared to maximum likelihood error (MLE). Currently CNNs are being used in the detection of COVID-19 patients through the classification of chest CT scans. In [31], a CNN based on multi-objective differential evolution (MODE) has been used to detect COVID-19 positive patients. In [32], a DeTraC (decompose, transfer, and compose) network has been developed by transfer learning a pre-trained ImageNet with the chest X-ray dataset for identification of COVID-19 patients. CNNs require huge data for training, and obtaining such an amount of data in a short duration is a major issue. In [33], an auxiliary classifier generative adversarial network (ACGAN) namely CovidGAN has been proposed to address the above issue. Training the CovidGAN was done via synthetic images of chest X-ray that were generated by the GAN. However, the recent work in [29-33] does not explore sparsity or noise in the measurements.

In the recent past, NLM filters have been employed to remove Rician noise from the MR images [5], however, sparsity inherent in the MR images was not exploited. Recently adaptive sparse reconstruction based on CNN (AsrCNN) was employed to explore patch based processing to denoise sparse images corrupted with Gaussian noise [34]. To our knowledge, CNN based approach to denoise sparse MR images corrupted with Rician noise has been scarcely explored. The proposed CNN based algorithm is capable of denoising the Rician noise corrupted sparse MR images and also reduces the computation time substantially. Hence, it has a potential applicability for online reconstruction. 


\section{Denoising Sparse MR Images via CNN-Mathematical Formulation}

In this section, we present the possible solutions to the sparse optimization problem. Initially, we discuss the existing Bayesian method for denoising the sparse MR image patches. Next, we consider optimization by rank minimization and develop the possible formulation with the CNN.

\subsection{Bayesian Approach}

Patch based processing of an image provides a better extraction of local features, hence in the present work, patch based reconstruction of an image has been emphasized. Let $a \in C^{m}$ be the vector representation of an image patch of dimension $\sqrt{m} \times \sqrt{m}$ to be reconstructed from very few $k$-space observations $b \in \complement^{n}$. To reconstruct the image patch, consider posterior distribution $X(a \mid b)$, which can be represented by Bayes' rule as

$$
X(a \mid b)=\frac{X(b \mid a) X(a)}{X(b)}
$$

Image reconstruction can be modelled by employing MAP estimation

$$
\arg \max _{a} X(a \mid b)=\arg \max _{a} X(b \mid a) X(a)
$$

applying log on both sides of the above equation:

$$
\arg \max _{a} \log X(a \mid b)=\arg \max _{a} \log X(b \mid a)+\log X(a)
$$

$\log X(a)$ and $\log X(a \mid b)$ represents the priori term and information probability respectively. Sparse recovery involves reconstruction of an image patch $a \in C^{m}$ from the acquired $k$-space measurements $b \in \complement^{n}$ such that, $n<m$ which is given by

$$
b=B a
$$

where $B \in C^{n \times m}$ is a measuring matrix, that encodes the undersampling operation, In the presence of noise, the measurements $b$ is given by

$$
b=B a+\sigma K
$$

here, $K$ represents the noise with standard deviation of $\sigma$. Further, the rank of the matrix $B a$ constitutes the dimension of the generated vector space, which signifies the sparsity. When a noise of Rician distribution interferes with the image, it introduces an image dependent bias. With Rician noise the objective of the proposed denoising algorithm to reconstruct an image patch $a$ can be rewritten from Eqs. (3) and (5) as,

$$
a=\arg \min _{a}\|b-B a\|_{2}^{2}+\sigma K(a)
$$

where, $K(a)$ represents the state linked with priori term $X(a)$. The estimated solution need to reduce both $l_{2}$ - norm problem and state parameter which is weighted by a factor $\sigma$ in the presence of noise. Hence, the term $\sigma K(a)$ plays a major role in reducing the noise present in the model. 


\subsection{Optimization Via Rank Minimization: a CNN Approach}

The problem of sparse coding can be effectively resolved by rank minimization method. The log-det (logarithm of determinant) function [35] minimizes the rank iteratively by finding the local minimum. In this section, we explore the usage of CNNs to iteratively reduce the value of log-det function and provide applicability in rank minimization.

The CNN framework utilizes the patch based processing for accurate reconstruction from sparse and noisy $k$-space measurements. Convolutional kernels in CNNs operate on extracted image patches by convoluting them with the kernel matrix. In the present paradigm it is assumed that the sparse signals/image patches possess self-similarities. At first image is converted into patches, and then the extracted patches are grouped to characterize the signal similarities. This technique reduces the optimization problem present in the various state-ofart-techniques. If $\mathbb{G}$ represents the set of all patches, considering the similarity and sparsity inherent in the MR images, various similar type of patches can be generated each of size $\sqrt{m} \times \sqrt{m}$ at position $f$ for $\hat{a}_{f} \in \mathbb{G}^{m}$. Let $C_{f}$ be the group of positions which corresponds to similar patches in an image, then $C_{f}$ can be expressed as

$$
C_{f}=\left[\left\{f_{k}\left\|\hat{\mathbb{a}}_{f}-\hat{\mathbb{a}}_{f_{k}}\right\|<H\right\}\right]
$$

where, $\hat{a}_{f}$ represents noiseless patches, $\hat{\mathrm{a}}_{f_{k}}$ are the corresponding noisy patches and the function $f_{k}$ minimizes the noise below a pre-defined threshold value $H$. Once grouping of patches is performed, a subsequent evaluation of information matrix can be done as $b=\left[b_{0}, b_{1}, \ldots b_{n-1}\right]$ and $C_{f} \in \mathbb{G}^{m \times n}$, where every column of $C_{f}$ represents a patch $a_{f}$. The present work assumes that all the patches in a batch possess same patterns. When the information matrix $b$ is corrupted with Rician noise the desired result changes. Reconstruction of MR image from less number of $k$-space measurements can be accomplished by rank optimization. The matrix rank for $B a$ can be recovered by sorting out the optimization issue as,

$$
B a=\arg \min _{B a} B a, \text { s.t. }\|b-B a\|_{2}^{2} \leq \lambda_{\sigma K}^{2}
$$

where $\|\cdot\|_{2}^{2}$ represents the L2 normalization and $\lambda_{\sigma K}^{2}$ represents the variance of Rician noise. The argument in Eq. (8) can be minimized by reducing the rank of matrix $\mathrm{Ba}$. Generally, rank minimization represents a NP-hard problem and it cannot be solved directly. The non-convex optimization methods provide an effective solution to rank minimization to achieve an efficient signal recovery [35]. The issue of rank optimization can be sorted out with the below equation, using logarithm of the determinant to provide smooth estimation for rank

$$
J(a, \delta)=\log \operatorname{det}(a+\delta I)
$$

here $\delta$ represents a small positive regularization constant. The function $\log \operatorname{det}(a+\delta I)$ is concave in $a$, hence its value decreases with each iteration, thereby converges rapidly to provide the right minimization. The function $J(a, \delta)$ evaluates the summation of logarithm of singular values that can be utilized as smooth surrogate for Rank $a$. The function $J(a, \delta)$ can provide better solution for rank optimization problem compared to other existing techniques. Hence, Eq. (9) can be rewritten as,

$$
J(B a, \delta)=\log \operatorname{det}\left(\left(B a B a^{T}\right)^{0.5}+\delta I\right)
$$




$$
\begin{gathered}
J(B a, \delta)=\log \operatorname{det}\left(Z D^{0.5} \frac{1}{Z}+\delta I\right) \\
J(B a, \delta)=\log \operatorname{det}\left(D^{0.5}+\delta I\right)
\end{gathered}
$$

where, $D$ represents a diagonal matrix and its diagonal elements can be defined as eigen values of matrix $B a B a^{T}$ which simplifies $B a B a^{T}=Z D \frac{1}{Z}$ and $D^{0.5}$ is the diagonal matrix with its diagonal elements defined as singular values of matrix $B a$. Then, the rank optimization problem to obtain the estimate $\hat{a}$ can be sorted as,

$$
\hat{a}=\arg \min _{B a} J(B a, \delta) \text { s.t. }\|b-B a\|_{2}^{2} \leq \lambda_{\sigma K}^{2}
$$

Estimating $\hat{a}$ in the presence of Rician noise through CNN training involves refining of weights $w$ with every iteration such that $\hat{a}=a$. Hence $a$ is updated based on the weights $w$ and can be formulated as

$$
(a, w)=\arg \min _{a, w}\|b-B a\|_{2}^{2}+\sigma K(w)
$$

\subsubsection{Proposed Formulation for Denoising}

This section presents the proposed CNN-based formulation for denoising and reconstruction of MR images from the sparse $k$-space data. The problem present in Eq. (14) can be solved further with CNNs by considering sparsity and iterative updation of weights to reduce the Rician noise. A possible formulation for reducing optimization problem is,

$$
(a, w)=\arg \min _{a, w}\|b-B a\|_{2}^{2}+\beta\left\|a-w^{(r)}\right\|_{2}^{2}+\sigma K(w) .
$$

The first term in the above equation handles sparsity, the second term reduces the noise iteratively and $\beta$ represents the regularization parameter. Dividing the optimization problem in Eq. (15) into two sub-problems (1) Estimating the image patches from the measurements (2) Denoising the patches iteratively by refining the weights through CNN training i.e.

$$
\begin{gathered}
a^{(r+1)}=\arg \min _{a}\|b-B a\|_{2}^{2}+\beta\left\|a-w^{(r)}\right\|_{2}^{2} \\
w^{(r+1)}=\arg \min _{w} \beta\left\|a^{(r+1)}-w\right\|_{2}^{2}+\sigma K(w)
\end{gathered}
$$

In the above equations $r$ represents the current iteration. An iterative gradient technique can be employed to estimate $a^{(r+1)}$, as finding inverse of the observation matrix $B$ can be inconvenient due to its large size. Then, the solution for $a$-subproblem of Eq. (16) can be given as

$$
a^{(r+1)}=a^{r}-\varphi\left[B^{T}\left(B a^{(r)}-b\right)+\beta\left(a^{(r)}-w^{(r)}\right)\right]
$$

here, $\varphi$ is defined as controlling factor for step-size. The term $B^{T}\left(B a^{(r)}-b\right)$ signifies minimizing the reconstruction error iteratively based on the feature maps and $\beta\left(a^{(r)}-w^{(r)}\right)$ minimizes the error by refining the weights.

This can be represented as, 


$$
a^{(r+1)}=\bar{B} a^{r}+\varphi B^{T} b+\varphi w^{(r)}
$$

where,

$$
\bar{B}=\left[(1-\varphi \beta) I-\varphi B^{T} B\right]
$$

The above equation indicates the updates of $a^{r}$ can be evaluated efficiently based on the computation of $\bar{B}$. The ideal solution of optimization problem is updation of $a^{r}$ to $a^{r+1}$. On the other hand, $w$ sub-problem can be evaluated at $a^{r+1}$ which is an operator of $K(w)$ and its solution can be obtained with the help of a denoiser as,

$$
w^{r+1}=f\left(a^{(r+1)}\right)
$$

where, $f(\cdot)$ denotes denoiser. Various techniques can be utilized to denoise the input MR images. In the present work, a CNN adopted from U-Net [21] is utilized to denoise huge training datasets. $\mathrm{CNN}$ based denoising is carried out by reducing the redundancies present in the noise corrupted images and also solves the optimization problem. We summarize the proposed method for denoising of MR images as given below

The observation matrix $B$ is initialized. The value of controlling factor $\varphi$ for step size and the regularization parameter $\beta$ is selected to be greater than zero and the pseudocode is summarized below

Choose the controlling factors $\varphi>0, \beta>0$ and compute $\bar{B}$ using Eq. (20)

1. Start with the iteration $r=0$

2. Initialize $a$ as $a^{(0)}=B^{T} b$; and $w^{(0)}=0$

3. do

$$
\begin{gathered}
a^{(r+1)}=\bar{B} a^{r}+\varphi B^{T} b+\varphi w^{(r)} \\
w^{r+1}=f\left(a^{(r+1)}\right)
\end{gathered}
$$

end while convergence is not achieved

4. Output: $a^{r+1}$

\section{CNN Architecture for Denoising}

This section describes the proposed denoising architecture based on the CNN model. The functional diagram of the proposed denoising algorithm is illustrated in Fig. 1. CNN is composed of two sections viz. encoding and decoding of features. Feature encoding, comprises of multiple convolutional layers followed by pooling layers. The reception of neurons can be enhanced with the help of pooling layers.

Convolutional layers in the encoding part employ kernels of size $3 \times 3$ with a stride of 1 . A non-linear activation ReLU was applied subsequently. Pooling layer is placed next to the convolutional layer to reduce the spatial resolution of the feature maps. The feature maps inside the pooling layer are convoluted with the kernels of size $2 \times 2$.

In the encoding stage, input is a $256 \times 256$ image which is followed by convolutional layers with 64-channel feature maps. A max pool layer is used next to downsample the feature maps to $128 \times 128$. The subsequent convolutional layers generate a 128-channel 


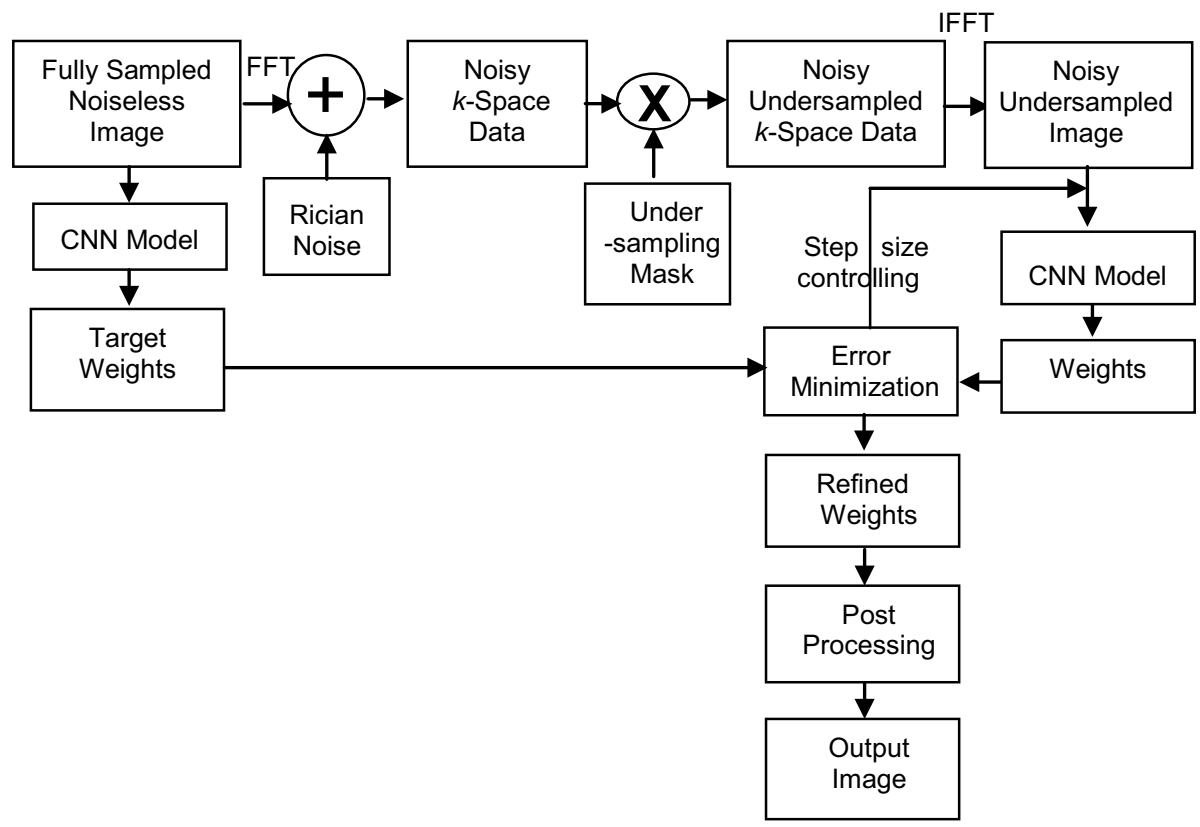

Fig. 1 Proposed CNN-based denoising model

feature maps. A max pool layer further downsamples the size of feature maps to $64 \times 64$. The lowest convolutional layer generates 256 channel feature maps. Encoding primarily doubles the number of feature map channels and at the same time reduces its size at every stage while traversing down the U-Net [21].

Decoding stage, also known as expansive path, involves upsampling of feature maps along with the deconvolution process. However, the reconstructed feature maps lose spatial information to large extent and the final reconstructed images may lose essential details of the images. Therefore, it is crucial to eliminate this problem and it can be achieved by fusing (concatenating) the feature maps from the encoder with the corresponding feature maps of the upsampling layer to generate updated and upsampled feature maps.

The decoding consists of convolutional layers with $3 \times 3$ kernels and a subsequent nonlinearity of $R e L U$ type, as in encoding. The 256 channel feature map obtained from the encoding is deconvolved to 128 channel feature maps. A max pool layer is placed after every convolution stage, to upsample the size of the feature maps. At every stage, the encoder feature maps from the same level are fused with the output of the convolutional layer. The final layer generates the desired number of classes to produce the reconstructed output image.

\subsection{Training Details}

The architecture of proposed CNN based denoising model is trained by an end-to-end process. Overfitting in the proposed $\mathrm{CNN}$ architecture has been reduced by employing drop out layer before the output layer. A learning rate of 0.001 has been employed and the momentum parameters have been fixed to $0.9\left(\beta_{1}\right)$ and $0.999\left(\beta_{2}\right)$. The value of 
epsilon was initialized to $1 \mathrm{e}-08$. CNN has been trained for 10,000 iterations with a batch size of 8. Proposed CNN uses Adam solver as it combines the advantage of AdaGrad and RMSProp [36]. Adam works with sparse and noisy gradients, moreover the parameter updates are invariant with the rescaling of gradients and provides a good control over the stepsize [36].

Input to the proposed algorithm is images normalized in the range $[0,1]$. Fully sampled, noiseless and normalized $256 \times 256$ images have been used to train the proposed CNN to obtain the target weights. A simulated Rician noise of level 10 is added to the same set of images in the $k$-space. Sparsity is achieved by applying $k$-space undersampling, from which around $75 \%$ samples are removed. Inverse Fourier transform converts the undersampled noisy $k$-space data to noisy and sparse MR images. These images are next given to the same CNN to generate observed weights which are further refined with error minimization. Target weights (obtained from fully sampled noiseless image) are considered as reference weights. CNN refines the weights by minimizing the loss function as given below

$$
\omega=\arg \min _{\omega} \sum_{f=1}^{M}\left\|\mathbb{F}\left(b_{f} ; \omega\right)-a_{f}\right\|_{2}^{2}
$$

where, $b_{f}$ and $a_{f}$ represents the $f^{\text {th }}$ pair of degraded and actual image patches respectively. $\mathbb{F}\left(b_{f} ; \omega\right)$ represents the patches of reconstructed image which consists of a parameter set as $\omega$. It is to be noted that the set of parameters $\omega$ is different from the weights $w$. The quality of reconstructed images can be further improved by employing several loss functions while training the CNN. Post processing involves scaling the normalized images back to the original intensity levels and dimensions. Figure 1 shows the schematic diagram of the proposed denoising model based on $\mathrm{CNN}$ and sparse $k$-space data.

\section{Performance Evaluation}

This section demonstrates the performance of the proposed algorithm by considering various images and undersampling schemes that include random, pseudoradial and Cartesian sampling. Experimentation has been performed with in vivo scans of MR images to evaluate the algorithm for undersampling limit. Experimental outcomes have been validated with various parameters namely PSNR [2], FSIM [37], HFEN [2] and QILV [38]. Our algorithm achieves high visual quality with detailed information and patterns in the reconstructed MR image.

\subsection{Implementation Details}

The CNN in the proposed algorithm has been trained on a GPU; it effectively removes Rician noise and improves the efficiency of MR data acquisition process. A parallel GPU configuration using CAFFE framework enhances the execution speed to a great extent. In the present work, every implementation has been carried out on an Intel i5 - 4460 processor with 16 GB RAM and 64-bit windows $10 \mathrm{OS}$ with $3.20 \mathrm{GHz}$ CPU. MATLAB 2016B has been used to simulate the results in this work. 


\subsection{Comparative Study}

The proposed CNN based denoising algorithm has been compared with various state-ofthe-art-techniques namely

(1) Dictionary learning magnetic resonance imaging (DLMRI) [2]

(2) Non-local means (NLM) and its variants namely unbiased NLM (UNLM), Rician NLM (RNLM), enhanced NLM (ENLM) and enhanced NLM filter with preprocessing (PENLM) [5].

Exclusive experiments were carried out on simulated $k$-space data of brain and in vivo $k$-space data of knee by increasing the undersampling limit from 4-fold to 20 -fold. All the experiments are validated not only by visual analysis but also by quantitative data assessment.

\subsubsection{Comparison with Dictionary Learning MRI}

This section presents the experimental results with the proposed CNN-based algorithm by considering axial brain, T2-weighted sagittal L-spine, C-spine images [39] and T1-weighted knee image [40]. The performance of the CNN-based reconstruction has been compared with DLMRI [2] and basic zero filled (ZF) reconstruction with respect to the PSNR and computation time. Rician noise level of around 10 has been added to the above reference images in the $k$-space and subsequently undersampled in the same domain. DLMRI iterations involve alternate sparse coding and dictionary learning stages. To implement DLMRI, Orthogonal Matching Pursuit (OMP) [41] was employed for sparse coding and 5 iterations of K-SVD [42] was used for learning the dictionary. Rest of the DLMRI parameters were chosen as in [39].

In Fig. 2, reconstruction with the proposed CNN-based method at 20-fold undersampling is presented and compared with the DLMRI and ZF reconstructions. Figure $2 \mathrm{a}$ depicts the 20-fold random undersampling having most of the samples in the central $k$-space. Figure $2 \mathrm{~b}$ shows the $512 \times 512$ axial brain image and Fig. $2 \mathrm{c}$ is the noise corrupted image. ZF reconstruction in Fig. 2d depicts prominent artifacts with $13.94 \mathrm{~dB}$ PSNR. DLMRI and CNN reconstruction for axial brain is given in Fig. 2e, f respectively. DLMRI reconstruction in Fig. 2e displays excessive smoothing and provides a PSNR of $26.12 \mathrm{~dB}$. CNN reconstruction removes noise effectively and reconstructs sharper image with $30.20 \mathrm{~dB}$ PSNR, which is $4.08 \mathrm{~dB}$ higher as compared to the DLMRI. Computation time with the proposed CNN was around $13 \mathrm{~s}$ whereas DLMRI needed nearly $60 \mathrm{~s}$ to reconstruct the same $512 \times 512$ axial brain image.

Figure 3 shows the reconstruction of $512 \times 512$ L-spine image with 4-fold Cartesian undersampling (Fig. 3a). Reference L-spine image is given in the Fig. $3 \mathrm{~b}$ and the noisy L-spine image is shown in Fig. 3c. ZF reconstruction in Fig. 3d displays some blurring due to $k$-space undersampling and its PSNR was observed to be $27.9 \mathrm{~dB}$. L-spine reconstructed with CNN method (Fig. 3f) provided a PSNR of $31.28 \mathrm{~dB}$ which was $2.08 \mathrm{~dB}$ higher than the PSNR with DLMRI $(29.25 \mathrm{~dB})$ in Fig. 3e. Computation time for CNN was around $13 \mathrm{~s}$ whereas DLMRI required around $70 \mathrm{~s}$.

Table 1 demonstrates the comparison of the quantitative results obtained with the proposed algorithm, DLMRI [2] and ZF reconstruction by 4-fold random undersampling of 


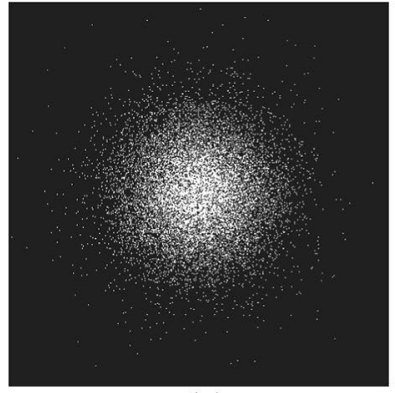

(a)

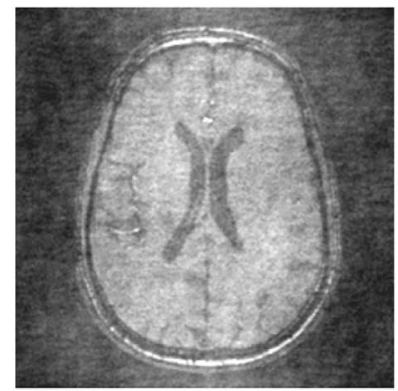

(d)

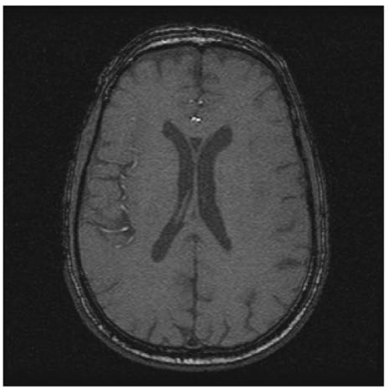

(b)

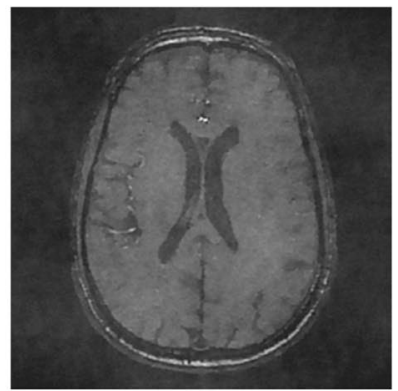

(e)

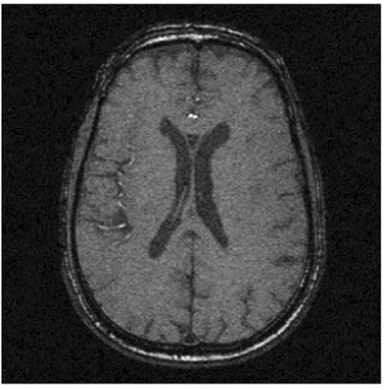

(c)

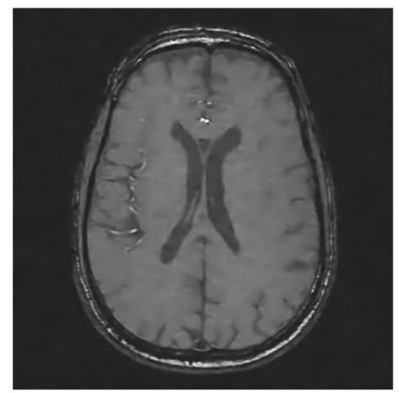

(f)

Fig. 2 Reconstructions with 20-fold random undersampling: a sampling mask, b reference axial brain image [39], c brain image with $10 \%$ Rician noise, d ZF reconstruction, e DLMRI reconstruction, f CNNbased reconstruction

the $k$-space (Fig. 4a). Experiments were carried out by considering the images of axial brain (Fig. 2b), L-spine (Fig. 3b), C-spine (Fig. 4b) and knee (Fig. 4c). Quantitative results with the proposed CNN method exhibit an improvement of around $2 \mathrm{~dB}$ in PSNR and a considerable reduction in the computation time, compared to DLMRI reconstruction. ZF reconstruction is obtained by applying inverse Fourier transform to the noisy and sparse $k$-space measurements. Hence, it provides very low PSNR and computation time.

\subsubsection{Comparison with Non-local Means}

This section illustrates the qualitative results obtained with our algorithm and also presents the quantitative comparison of the proposed $\mathrm{CNN}$-based denoising with the variants of NLM [5]. MR sequences like T1, T2 and Proton Density (PD)-weighted provides excellent contrast and anatomical details; however, under the influence of Rician noise the image quality deteriorates. Rician noise introduces bias that leads to the blurring of edges and structural errors. Images from BrainWeb Database [43] have been employed for simulations in this section. Experiments have been carried out on T1-weighted (Fig. 5a), T2-weighted (Fig. 5d) and PD-weighted (Fig. 5g) MR images, to evaluate the performance of the CNN-based proposed algorithm. All the parameters have been selected according to the requirement and a Rician noise of around $9 \%$ has been added to the images in the $k$-space. 


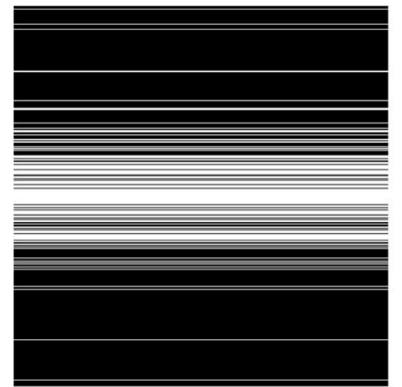

(a)

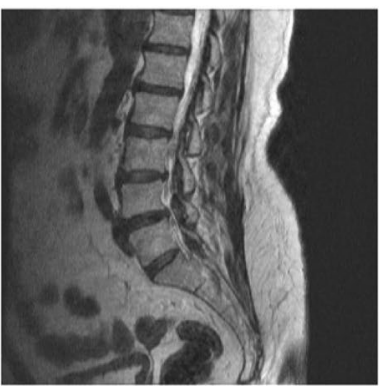

(d)

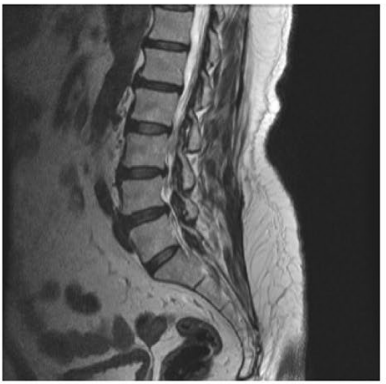

(b)

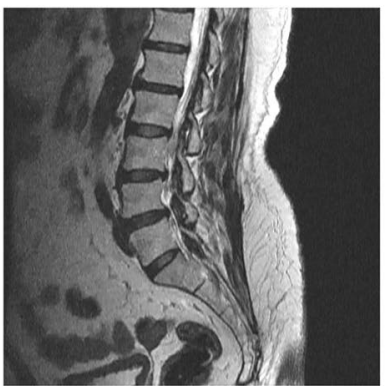

(e)

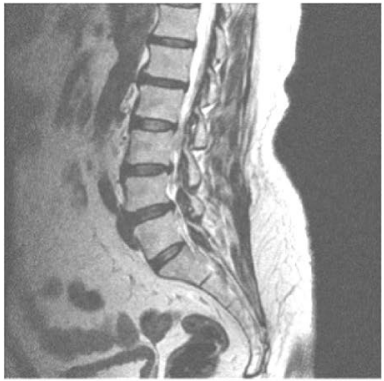

(c)

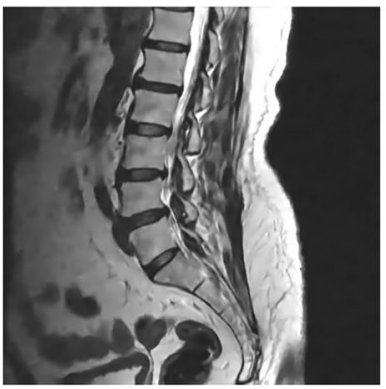

(f)

Fig. 3 Reconstructions with 4-fold Cartesian undersampling a sampling mask, b reference image of T2-weighted L-spine, c L-spine with $10 \%$ Rician noise, d ZF reconstruction, e DLMRI reconstruction, $\mathbf{f}$ CNN-based reconstruction

Table 1 Comparison of PSNR and computation time with 4-fold random undersampling

\begin{tabular}{lllrrr}
\hline Algorithm & Metrics & \multicolumn{2}{l}{ Images } & & \\
\cline { 3 - 6 } & & C-Spine & L-Spine & Knee & Brain \\
\hline ZF & PSNR (dB) & 24.1 & 24.67 & 25.32 & 23.49 \\
\multirow{2}{*}{ DLMRI } & Time (s) & 1.11 & 1.67 & 1.04 & 0.77 \\
& PSNR (dB) & 30.57 & 29.53 & 31.53 & 28.79 \\
Proposed CNN based & Time (s) & 75.91 & 73.17 & 44.33 & 62.4 \\
& PSNR (dB) & 32.32 & 31.97 & 33.19 & 30.19 \\
& Time (s) & 13.7 & 12.6 & 13 & 13.8 \\
\hline
\end{tabular}

Figure 5 presents the visual results with the proposed algorithm for 4-fold undersampling of the $k$-space by considering the above MR image sequences. Our algorithm denoises and provides accurate reconstruction of T1-weighted image with a PSNR of $25.55 \mathrm{~dB}$ in Fig. 5c. PSNR of the reconstructed T2-weighted image in Fig. $5 \mathrm{f}$ is $30.53 \mathrm{~dB}$ and displays good denoising. A PSNR of $31.29 \mathrm{~dB}$ was observed with the reconstructed PD-weighted image (Fig. 5i). Reconstruction time for our algorithm was observed to be few seconds.

Table 2 compares the numerical results of the proposed method with the NLM and its variants. The proposed CNN-based method has been implemented with a 4-fold and 


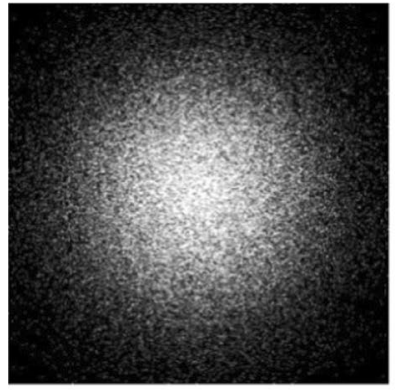

(a)

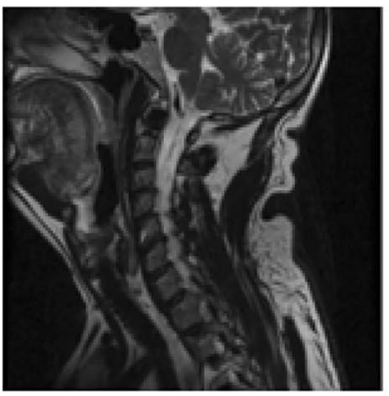

(b)

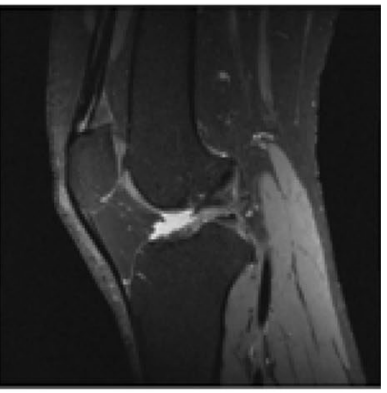

(c)

Fig. 4 Random sampling and reference images a 4-fold random undersampling mask, b T2-weighted C-spine, c T1-weighted knee

20-fold undersampling of the $k$-space unlike NLM, which considers entire $k$-space data for reconstruction. Quantitative outputs confirms that the CNN based denoising could achieve better PSNR for T2 and PD-weighted compared to NLM, UNLM and RNLM even with 20 -fold undersampling. Standard deviation (SD) of the Rician noise for NLM and the proposed method has also been displayed in Table 2. The numerical values obtained with the reconstructions of Fig. 5 are indicated in bold as shown in Table 2.

Visual quality of the reconstructions manifests that the proposed denoising algorithm has the capacity to effectively remove the Rician noise as well as reconstruct edges precisely even with 4-fold undersampling.

\subsubsection{Analysis of Undersampling Limit and Schemes}

Dedicated experiments were carried out with the random and pseudoradial sampling scheme to test the proposed CNN-based denoising algorithm for achievable undersampling limit. A complex Rician noise of $10 \%$ was added in the $k$-space to the $256 \times 256$ ground truth image of brain (Fig. 6a) and $320 \times 320$ human knee image (Fig. 6b) from the dataset of [44]. The random undersampling of Fig. 4a was increased from 4-fold to 20-fold for the experimentation to record the PSNR, FSIM, QILV and runtime variations, as given in Table 3. Proposed algorithm reconstructs MR images with precision even at undersampling rates as high as 20-fold, which is evident from the high PSNR, FSIM and QILV values. Computation time to reconstruct brain and knee image is between 3 to $6 \mathrm{~s}$. PSNR was observed to be consistent even at high undersampling rates and with various sampling patterns like pseudoradial. The minimum reconstruction time observed for ground truth brain and in vivo knee images are marked in bold as shown in Table 3.

The algorithm was also evaluated with various undersampling schemes by considering the reference images of Fig. 6 corrupted with the same noise level as above.

A structural undersampling was adopted with a pseudoradial sampling mask as demonstrated in Fig. 7a, which contains 30\% $k$-space samples. Figure $7 \mathrm{~b}$ displays the noise corrupted brain image obtained by adding $10 \%$ Rician noise to the reference image of Fig. 6a. The reconstructed brain image displayed in Fig. 7c provided a PSNR of $28.32 \mathrm{~dB}$, similarity index (FSIM) and local variance (QILV) was observed to be 0.966 and 0.9677 respectively. 


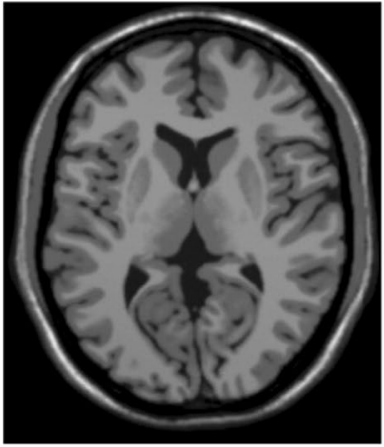

(a)

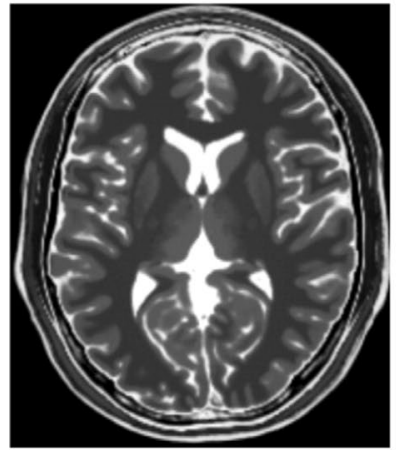

(d)

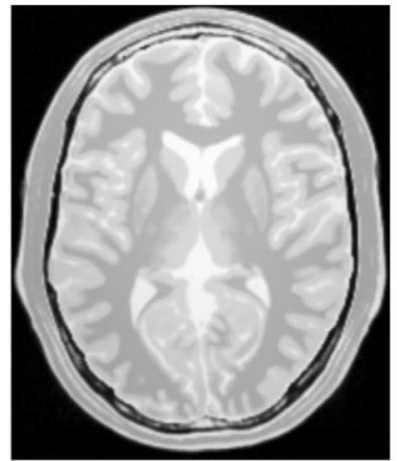

(g)

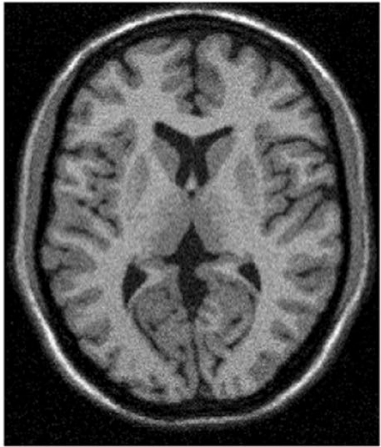

(b)

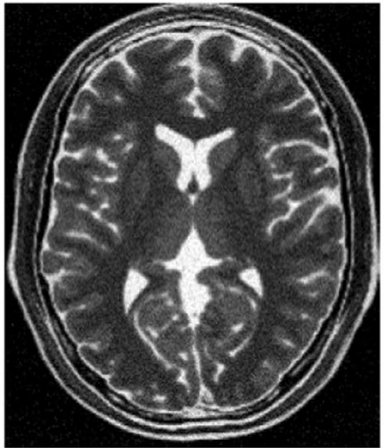

(e)

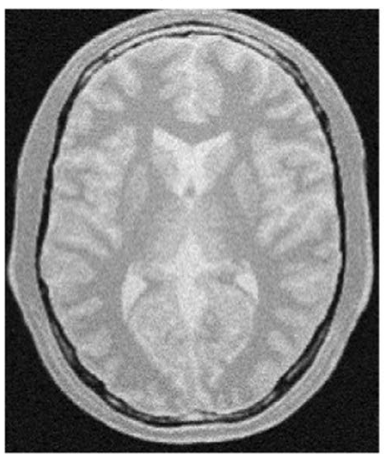

(h)

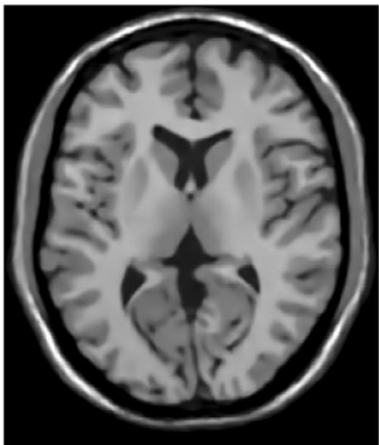

(c)

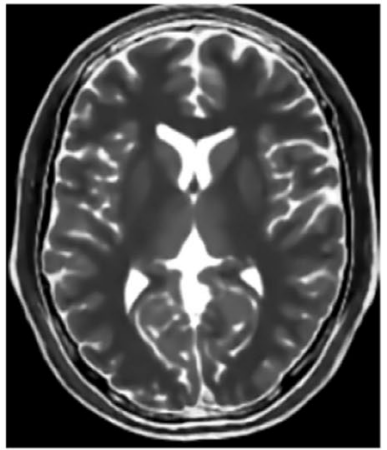

(f)

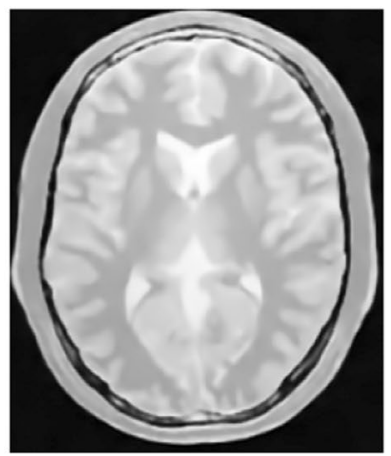

(i)

Fig. 5 CNN-based reconstructions at 4-fold random undersampling. a T1-weighted reference brain image [5], b T1 image with 9\% Rician noise, c Reconstructed T1 image, d T2-weighted reference brain image [5], e T2 image with 9\% Rician noise, f reconstructed T2 image, $\mathbf{g}$ PD-weighted reference brain image [5], h PD image with $9 \%$ Rician noise, $\mathbf{i}$ reconstructed PD image

Next, in vivo knee MR image of Fig. 6b was undersampled with Cartesian sampling mask (Fig. 7d) obtained by acquiring $50 \%$ of the $k$-space points. Rician noise corrupted image, prior to undersampling is shown in Fig. 7e and the reconstructed knee image with 
Table 2 Numerical results for T1, T2 and PD-weighted images [5, 43] with respect to PSNR and Undersampling

\begin{tabular}{lllll}
\hline MR image $\rightarrow$ & T1- weighted & T2- weighted & PD- weighted & $\begin{array}{l}\text { Undersampling } \\
\text { employed }\end{array}$ \\
\hline Algorithm & PSNR (dB) & PSNR (dB) & PSNR (dB) & No \\
NLM & 28.91 & 24.49 & 24.72 & No \\
UNLM & 33.18 & 27.46 & 29.18 & No \\
RNLM & 32.79 & 28.64 & 28.83 & No \\
ENLM & 35.06 & 30.29 & 31.54 & No \\
PENLM & 36.84 & 31.76 & 32.76 & 20-Fold \\
Proposed CNN-based & 24.82 & 30.04 & 30.89 & 4-Fold \\
Proposed CNN-based & $\mathbf{2 5 . 5 5}$ & $\mathbf{3 0 . 5 3}$ & $\mathbf{3 1 . 2 9}$ & No \\
SD-NLM & 13.5 & 22.5 & 22.95 & Yes \\
SD :proposed CNN-based & 0.23 & 0.23 & 0.32 &
\end{tabular}

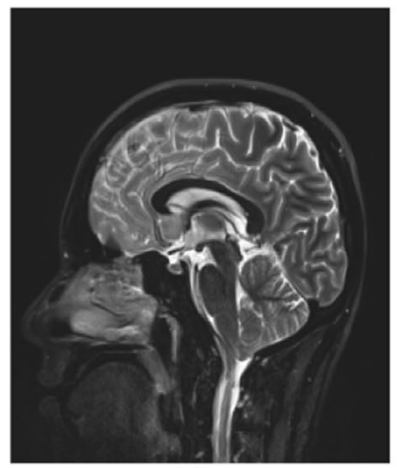

(a)

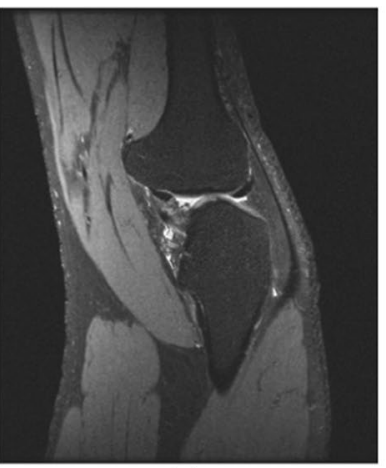

(b)

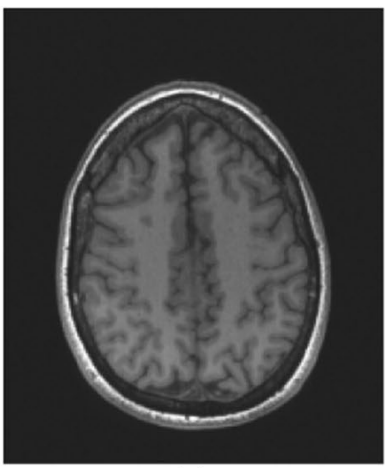

(c)

Fig. 6 Reference images [44] a ground truth brain, b In vivo knee, c In vivo axial brain

Table 3 Quantitative results with the proposed algorithm for brain image (Fig. 6a) and in vivo knee MR image (Fig. 6b)

\begin{tabular}{llllll}
\hline Sampling scheme & Image & PSNR in dB & FSIM & QILV & Time (s) \\
\hline Random-4 fold & Brain & 28.88 & 0.97 & 0.97 & 3.8 \\
& Knee & 31.88 & 0.96 & 0.76 & 5.3 \\
Random-6 fold & Brain & 28.63 & 0.97 & 0.97 & $\mathbf{3 . 5}$ \\
& Knee & 31.78 & 0.96 & 0.75 & 5.3 \\
Random-8 fold & Brain & 28.37 & 0.97 & 0.97 & $\mathbf{3 . 5}$ \\
& Knee & 31.72 & 0.96 & 0.75 & 5.3 \\
Random-10 fold & Brain & 28.30 & 0.97 & 0.97 & 3.7 \\
& Knee & 31.71 & 0.95 & 0.74 & $\mathbf{5 . 1}$ \\
Random-20 fold & Brain & 28.32 & 0.97 & 0.97 & 3.6 \\
& Knee & 31.72 & 0.96 & 0.74 & 6.1 \\
Pseudo Radial-7 fold & Brain & 28.48 & 0.97 & 0.97 & 3.6 \\
& Knee & 31.73 & 0.96 & 0.75 & 5.3 \\
\hline
\end{tabular}




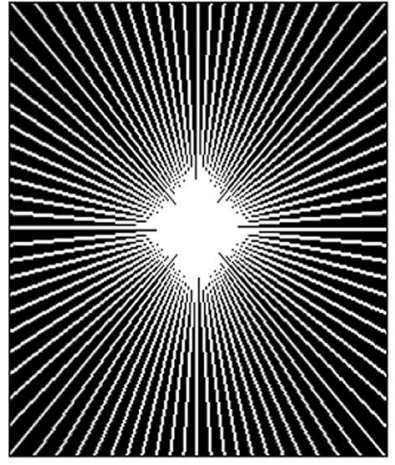

(a)

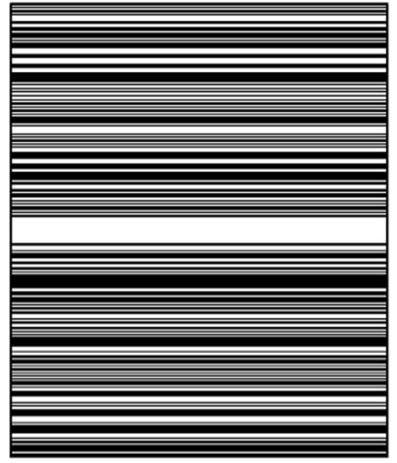

(d)

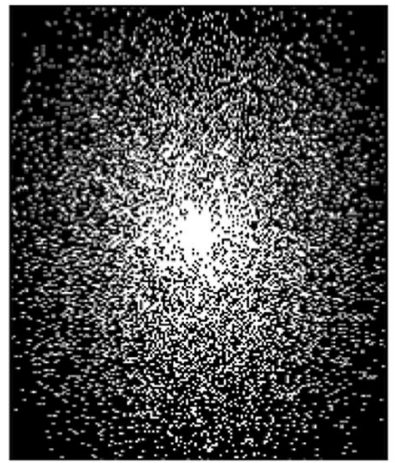

(g)

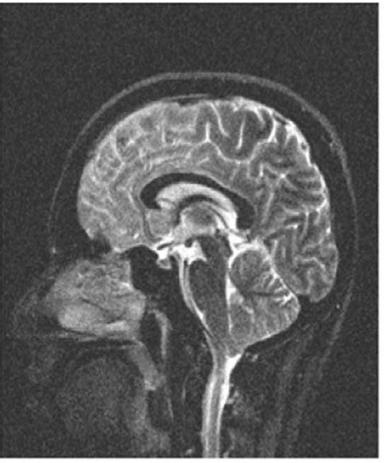

(b)

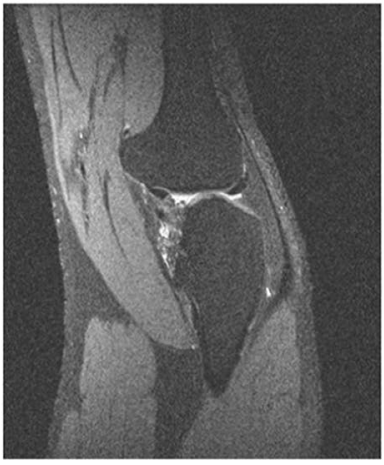

(e)

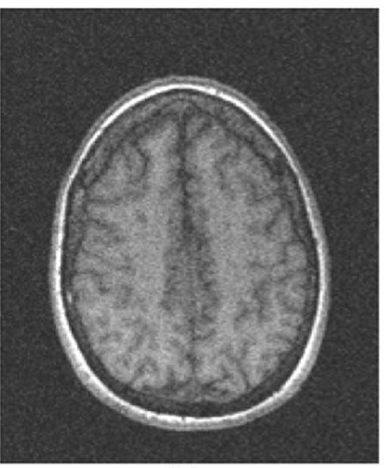

(h)

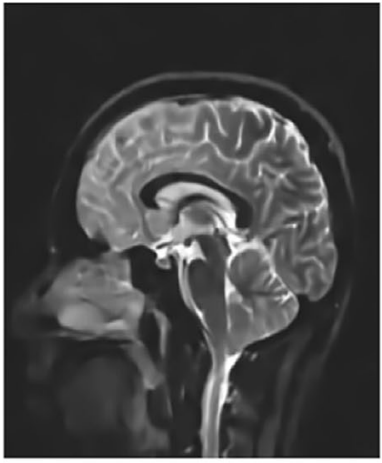

(c)

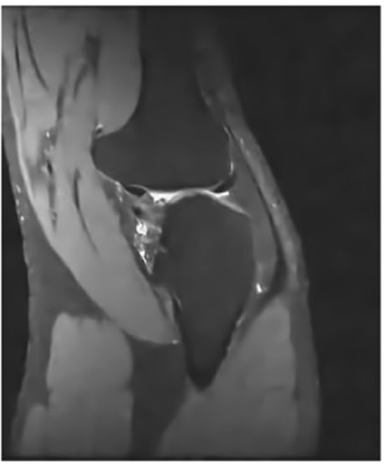

(f)

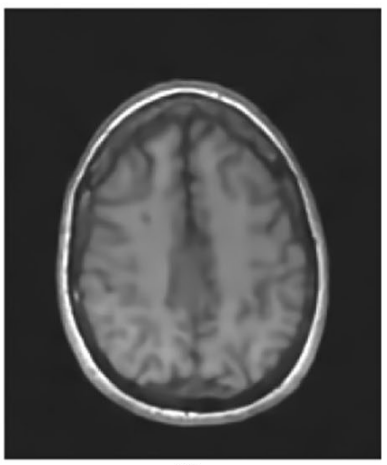

(i)

Fig. 7 Visual representation of the reconstructed MR images using the proposed CNN-based denoising algorithm with $10 \%$ Rician noise added to the reference images of Fig. 6. a Pseudoradial mask [44], b noisy brain image, $\mathbf{c}$ reconstructed brain image, $\mathbf{d}$ Cartesian mask [44], e noisy knee image, $\mathbf{f}$ reconstructed knee image, $\mathbf{g}$ random mask [44], $\mathbf{h}$ noisy axial brain image, $\mathbf{i}$ denoised axial brain image

the proposed CNN-based method is presented in Fig. 7f. A PSNR of $31.58 \mathrm{~dB}$ has been observed with the reconstructed knee image. QILV and FSIM were noted to be 0.7428 and 0.9548 respectively. 
We consider a $230 \times 180$ axial image of brain shown in Fig. 6c as the final image for the experimentation. This image was undersampled in $k$-space with $20 \%$ randomly acquired sampling pattern of Fig. $7 \mathrm{~g}$. A noise corrupted version of the axial brain image is given in Fig. 7h. Proposed algorithm reconstructs the denoised image (Fig. 7i) with a PSNR of $27.47 \mathrm{~dB}$. QILV and FSIM values were observed to be 0.9897 and 0.9644 respectively.

Experimental results with proposed algorithm show high quality reconstructions even with $80 \%$ missing $k$-space data. PSNR decreased by about $0.5 \mathrm{~dB}$ as the random undersampling rate was increased from 4-fold to 20-fold for brain image (Table 3) and maximum reconstruction time is $3.8 \mathrm{~s}$. Quantitative results in Table 3 show only $0.2 \mathrm{~dB}$ variation in PSNR for knee image and maximum reconstruction time is as low as $5.1 \mathrm{~s}$. Low computational time makes the proposed $\mathrm{CNN}$ based algorithm suitable for the online reconstruction.

\section{Conclusion}

In this manuscript, a CNN based novel framework to denoise sparse MR images corrupted with Rician noise, has been presented. CNN training exploits patch based processing to update and refine the dictionary of weights. Training eliminates the Rician noise and also estimates the missing $k$-space data to provide precise reconstructions even at high undersampling rates. The proposed CNN based denoising method converges faster and extracts MR image patterns in the recovered image, thereby boosting the speed and efficiency of MR data acquisitions. Our CNN based method has been compared with DLMRI and ZF reconstruction in terms of PSNR and computation time. There has been a tremendous increase in speed and PSNR due to CNN based approach when compared to DLMRI and ZF.

The proposed CNN framework can be employed without estimating the noise level, further our algorithm preserves the local structures better than the traditional NLM, UNLM and RNLM even with high undersampling. The computation time using our algorithm is very low, minimum being $3.5 \mathrm{~s}$. Proposed algorithm reconstructs with high visual quality even at high undersampling rates which is evident from the PSNR, FSIM and QILV values.

High undersampling rates provide required compression of $k$-space data the acquisition stage and can be employed for wireless transmission. However, various issues like bandwidth requirement, power consumption and encoding, associated with wireless streaming of $k$-space data needs to be addressed and remains the subject of future study. The CNN approach can be adopted for image segmentation to identify COVID-19 patients efficiently to overcome the current crisis.

\section{References}

1. Lustig, M., Donoho, D. L., \& Pauly, J. M. (2007). Sparse MRI: The application of compressed sensing for rapid MR imaging. Magnetic Resonance in Medicine, 58, 1182-1195.

2. Ravishankar, S., \& Bresler, Y. (2011). MR image reconstruction from highly undersampled $k$-space data by dictionary learning. IEEE Transactions on Medical Imaging, 30, 1028-1041.

3. Le Pennec, E., \& Mallat, S. (2005). Sparse geometric image representations with bandelets. IEEE Transactions on Image Processing, 14, 423-438. 
4. Jang, U., et al. (2013). Improvement of the SNR and resolution of susceptibility weighted venography by model-based multi-echo denoising. NeuroImage, 70, 308-316.

5. Hong, L., et al. (2010). Denoising 3D MR images by the enhanced non-local means filter for Rician noise. Magnetic Resonance Imaging, 28, 1485-1496.

6. Chunli, W., Xiaowan, L., et al. (2017). An improved total variation regularized SENSE reconstruction for MRI Images. In 29th Chinese control and decision conference (CCDC), Chongqing, pp. 5005-5009.

7. Yang, A. C., et al. (2016). Sparse reconstruction techniques in MRI: Methods, applications, and challenges to clinical adoption. Investigative Radiology, 51(6), 349-364.

8. Mrinmoy, S., \& Nirmala, S. R. (2017). Compressed sensing trends in magnetic resonance imaging. Engineering Science and Technology, an International Journal, 20(4), 1342-1352.

9. Renna, F., et al. (2014). Reconstruction of signals drawn from a gaussian mixture via noisy compressive measurements. IEEE Transactions on Signal Processing, 62, 2265-2277.

10. Chang, H., Li, C., \& Gallogly, A. H. (2018). Brain MR image restoration using an automatic trilateral filter with GPU-based acceleration. IEEE Transactions on Biomedical Engineering, 65(2), 400-413.

11. Hu, L., Shengrong, Z., \& Xiangjun, D. (2017). The brain MRI image sparse representation based on the gradient information and the non-symmetry and anti-packing model. Computer Assisted Surgery Journal, 22(2), 106-112.

12. Hákon, G., \& Samuel, P. (1995). The Rician distribution of noisy MRI data. Magnetic Resonance in Medicine, 34(6), 910-914.

13. Knoll, F., Bredies, K., Pock, T., \& Stollberger, R. (2011). Second order total generalized variation (TGV) for MRI. Magnetic Resonance in Medicine, 65, 480-491.

14. Haldar, J. (2014). Low-rank modeling of local $k$-space neighborhoods (LORAKS) for constrained MRI. IEEE Transactions on Medical Imaging, 33, 668-681.

15. Yuxin, Hu, et al. (2019). Motion robust reconstruction of multi-shot diffusion-weighted images without phase estimation through locally low-rank regularization. Magnetic Resonance in Medicine, 81(2), 1181-1190.

16. Krizhevsky, A. (2012). Imagenet classification with deep convolutional neural networks. Advances in Neural Information Processing Systems, 25, 1097-1105.

17. He, K., et al. (2016). Deep residual learning for image recognition. In 2016 IEEE conference on computer vision and pattern recognition (CVPR), Las Vegas, NV, pp. 770-778. https://doi.org/10.1109/ cvpr.2016.90.

18. Schlemper, J., et al. (2018). Stochastic deep compressive sensing for the reconstruction of diffusion tensor cardiac MRI. Computer Vision and Pattern Recognition, arXiv:1805.12064.

19. Maximilian S., et al. (2018). adversarial and perceptual refinement for compressed sensing MRI reconstruction. Computer Vision and Pattern Recognition. arXiv:1806.11216v1.

20. Yoseob, H., Jaejun, Y., et al. (2018). Deep learning with domain adaptation for accelerated projectionreconstruction MR. Computer Vision and Pattern Recognition. arXiv:1703.01135v2.

21. Chang, M. H., et al. (2018). Deep learning for undersampled MRI reconstruction. Physics in Medicine and Biology, 63, 135007.

22. Yoseob, H., \& Jong, C. Y. (2018). Framing U-Net via deep convolutional framelets: Application to sparse-view CT. IEEE Transactions on Medical Imaging, 37(6), 1418-1429.

23. Zongwei, Z. (2018). UNet++: A nested U-Net architecture for medical image segmentation. arXiv :1807.10165v1.

24. Zongwei, Z., et al. (2019). UNet++: redesigning skip connections to exploit multiscale features in image segmentation. IEEE Transactions on Medical Imaging. https://doi.org/10.1109/TMI.2019.29596 09.

25. Nguyen-Duc, T., \& Jeong, W. (2018). Compressed sensing dynamic MRI reconstruction using multiscale 3D convolutional sparse coding with elastic net regularization. In 2018 IEEE 15th international symposium on biomedical imaging (ISBI 2018), Washington, DC, pp. 332-335.

26. Joseph, Y., Cheng, F., et al. (2018). Highly scalable image reconstruction using deep neural networks with bandpass filtering. Computer Vision and Pattern Recognition. arXiv:1805.03300.

27. Kerem, C., Tezcan, et al. (2018). MR image reconstruction using deep density priors. Compueter vision and pattern recognition. arXiv:1711.11386v3.

28. Lee, D., Yoo, J., \& Ye, J. C., (2017). Deep residual learning for compressed sensing MRI. In IEEE 14th international symposium on biomedical imaging (ISBI 2017), Melbourne, VIC, pp. 15-18.

29. Medley, D. O., et al. (2019). Segmenting the left ventricle in cardiac in cardiac MRI: From handcrafted to deep region based descriptors. In 2019 IEEE 16th international symposium on biomedical imaging, Venice, Italy, pp. 644-648. 
30. Ribeiro S. E. (2019). A deep learning approach to T1 mapping in quantitative MRI. In 36th annual scientific meeting of the European society for magnetic resonance in medicine and biology, Rotterdam, The Netherlands, vol. 32 (Suppl. 1). Magnetic resonance materials in physics.

31. Dilbag, S., et al. (2020). Classification of COVID-19 patients from chest ct images using multi-objective differential evolution-based convolutional neural networks. European Journal of Clinical Microbiology and Infectious Diseases. https://doi.org/10.1007/s10096-020-03901-z.

32. Abbas, A., et al. (2020). Classification of COVID-19 in chest X-ray Images using DeTraC deep convolutional neural network. medRxiv preprint doi: https://doi.org/10.1101/2020.03.30.20047456.

33. Waheed, A., et al. (2020). CovidGAN: Data augmentation using auxiliary classifier GAN for improved Covid-19 detection. IEEE Access, 8, 91916-91923. https://doi.org/10.1109/ACCESS.2020.2994762.

34. Manimala, M. V. R., Dhanunjaya Naidu, C., \& Giri Prasad, M. N. (2020). Convolutional neural network for sparse reconstruction of MR images interposed with gaussian noise. Journal of Circuits, Systems, and Computers, 29(7), 2050116. https://doi.org/10.1142/S0218126620501169.

35. Fazel, M., Hindi, H., \& Boyd, S. (2004). Rank minimization and applications in system theory. In proceeding of the 2004 American control conference, Boston, Massachusetts.

36. Kingma, D. P., Ba, J. (2015). Adam: A method for stochastic optimization. In International conference on learning representations (ICLR-2015).

37. Zhang, L., et al. (2011). FSIM: a feature similarity index for image quality assessment. IEEE Transactions on Image Processing, 20, 2378-2386.

38. Aja-Fern'andez S., et al. (2006). Image quality assessment based on local variance. In 28th Conf. Proc. IEEE Eng. Med. Biol. Soc., pp. 4815-4818.

39. Manimala, M. V. R., Dhanunjaya Naidu, C., \& Giri Prasad, M. N. (2018). Convex optimization and greedy iterative algorithms for dictionary learning in the presence of Rician noise. International Journal of Engineering and Technology, 7(4), 4922-4929.

40. mridata.org

41. Tropp, J., \& Gilbert, A. C. (2007). Signal recovery from random measurements via orthogonal matching pursuit. IEEE Transactions on Information Theory, 53(12), 4655-4666.

42. Aharon, M., Elad, M., \& Bruckstein, A. (2006). K-SVD: An algorithm for designing overcomplete dictionaries for sparse representation. IEEE Transactions on Signal Processing, 54(11), 4311-4322.

43. http://www.bic.mni.mcgill.ca/brainweb/

44. Ramos-Llordén, G., den Dekker, A. J., \& Sijbers, J. (2017). Partial discreteness: A novel prior for magnetic resonance image reconstruction. IEEE Transactions on Medical Imaging, 36(5), 1041-1053.

Publisher's Note Springer Nature remains neutral with regard to jurisdictional claims in published maps and institutional affiliations.

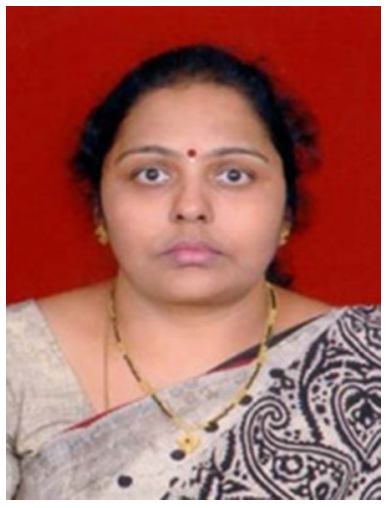

M. V. R. Manimala has received B.E. from the Department of Electronics and Communication, C.R.R. College of Engineering, Andhra University in 1994 and M.Tech from JNTUH, in 2003. She is pursuing her Ph.D from JNTUA, Ananthapuramu. She has been working at M.V.S.R. Engineering College since 2000, and is currently serving as Associate Professor in the Department of Electronics and Communication Engineering. 


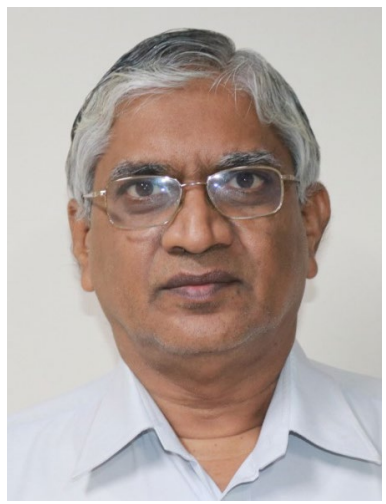

Dr. C. Dhanunjaya Naidu received B.Tech from the Department of Electronics and Communication, JNTUA in 1982 and M.Tech in Instrumentation and Control Systems from S.V. University in 1985 . He was awarded Ph.D degree in Image Processing with Neural Networks from JNTUH in 2002. In 1997, he joined VNRVJIET and is currently the Principal of the college. He has also served in the capacity of VicePrincipal, Dean-Academics, Professor and Head-ECE Department at VNRVJIET. He was formerly associated with Vasavi College of Engineering, Hyderabad and KSRM College of Engg. Kadapa, A. P. His research areas include Digital signal processing, Digital filters, Neural Networks, Wavelet Transforms and Image Processing and Analysis.

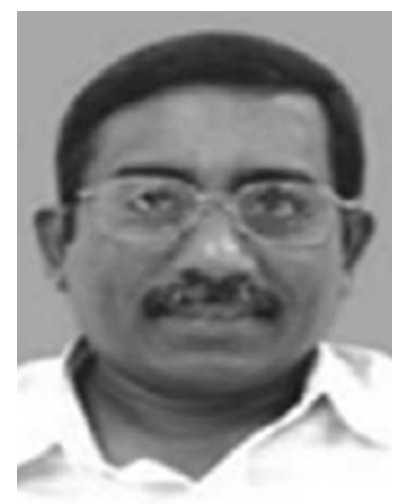

Dr. M. N. Giri Prasad received the B.Tech degree in ECE from JNTUA and M.Tech from S.V. University, Tirupati in 1982 and 1994 respectively. He received Ph.D. degree from JNTUH, Hyderabad, in 2003. $\mathrm{He}$ is currently a Professor with ECE Department and Director of Admissions, JNTUA, Ananthapuramu, India. His areas of interests are biomedical signal processing, image processing, communications and embedded systems. He is a member in professional societies like the ISTE, the IEI, and the NAFEN. 